\title{
DISCOVERING COMMUNITIES FROM SOCIAL NETWORKS: METHODOLOGIES AND APPLICATIONS
}

Bo Yang, Dayou Liu and Jiming Liu

\section{Introduction}

A network community refers to a group of network nodes within which the links connecting them are dense but between which they are sparse. A network community mining problem (NCMP for short) can be stated as the problem of finding all such communities from a given network. A large variety of problems can be translated into NCMPs, ranging from graph partition applications, such as VLSI layout $[1,2]$, large-scale scientific computing [3, 4], load balance for distributed computing [5, 6], and image segmentation [19], to complex network analysis, such as social network analysis [7, 8, 9], biological network analysis[11, 12, 24], and Web pages clustering $[13,14,15]$.

Network communities in different application contexts may imply different meanings and serve different purposes. For example, they may be sets of electronic units closely placed on a VLSI circuit board, collections of processes that frequently communicate with each other, segments of an image, circles of a society within which people share common interests and keep frequent contacts, classes of Web pages related to common topics, or groups of proteins exhibiting similar functions. Discovering such hidden patterns from networks will enable us to better understand the structural and/or functional characteristics of networks and to more effectively utilize them.

The remainder of this chapter is organized as follows: Section 2 introduces some of the existing methods for solving NCMPs. Section 3 presents several interesting applications of NCMPs as related to social network analysis. Finally, Section 4 concludes the chapter.

Bo Yang, Dayou Liu

School of Computer Science and Technology, Jilin University, China. e-mail: ybo@jlu.edu.cn

Jiming Liu

Department of Computer Science, Hong Kong Baptist University, Hong Kong. e-mail: jiming@comp.hkbu.edu.hk 


\section{Methodologies of network community mining}

In view of the basic strategies adopted, most of the existing community mining algorithms can be classified into two main categories: optimization based algorithms and heuristic based algorithms. The former solves an NCMP by transforming it into an optimization problem and trying to find an optimal solution with respect to a pre-defined objective function, such as various cut criteria adopted by spectral methods $[16,17,18,19,2,20]$, the evaluation function introduced by the Kernighan-Lin algorithm [21], the network modularity employed in several algorithms [22, 23, 24, 25, 26] and others [27]. On the contrary, heuristic algorithms do not explicitly state optimization objectives, and they solve an NCMP based on certain intuitive assumptions or heuristic rules. For example, the heuristic rule used in the maximum flow community (MFC) algorithm [14] is based on the assumption that "flows" through inter-community links should be larger than those of intracommunity links. Similarly, the heuristic rule employed by the GN algorithm [7] is that the the "edge betweenness" of inter-community links should be larger than that of intra-community links. Others such as the Wu-Huberman algorithm [28], the HITS algorithm [13], the CPM[29], and the FEC [30] have adopted different assumptions.

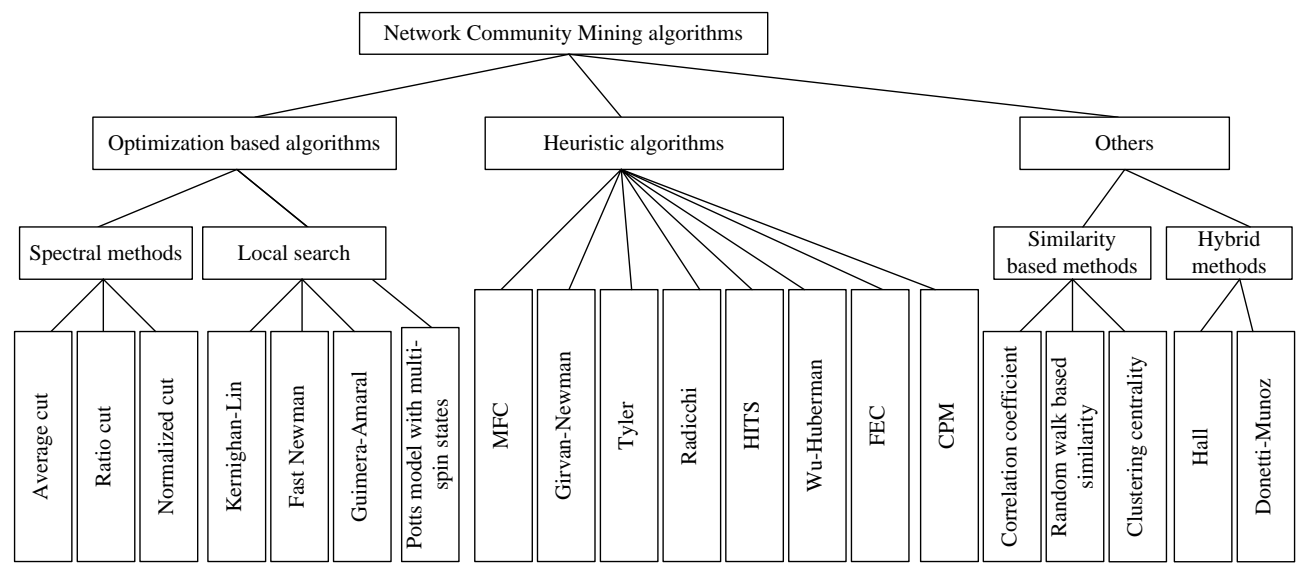

Fig. 1 The Classification chart for some of the existing community mining algorithms. [47]

\subsection{Optimization based algorithms}

Spectral methods and local search based methods are two representatives of optimization based algorithms for solving NCMPs. 
Spectral methods optimize certain pre-defined cut criteria by using the quadratic optimization technique. In graph theory, the cut of a bipartition of a network is defined as the number of inter-group links. An optimal bipartition of a network is the one with the minimum cut. Yet, in most cases, the minimum cut criterion will lead to bias partitions. In order to avoid this problem, other criteria, such as average cut [16, 17], ratio cut [20], normalized cut [19], and their variants, have been proposed to compute the density, instead of the number, of inter-group links. Unfortunately, the problems of finding different optimal cuts have been proven to be NP-complete $[31,19]$. Based on matrix theory, the spectral methods try to find an approximately optimal cut by transforming the problem into a constraint quadratic optimization problem represented as $\min \left(X^{T} M X\right) /\left(X^{T} X\right)$, where $X$ denotes the indicator vector of a bipartition, and $M$ is a symmetric positive semi-definite matrix. In the case of minimizing the average cut, $M$ corresponds to the Laplacian matrix of a given graph. In the case of minimizing the ratio cut, normalized cut, or others, $M$ corresponds to a variant of the Laplacian matrix. Thus, an approximately optimal solution of the constraint quadratic optimization problem can be obtained by means of calculating the second smallest eigenvector of $M$.

Generally speaking, computing all eigenvectors of a matrix will take $O\left(n^{3}\right)$ time, where $n$ is the number of nodes. While in a sparse matrix, the second smallest eigenvector can be calculated with the time complexity of $O\left(m /\left(\lambda_{2}-\lambda_{3}\right)\right)$ by applying the Lanczos method. Here, $m$ denotes the number of links in a network, and $\lambda_{2}$ and $\lambda_{3}$ denote the second and third smallest eigenvalues of $M$, respectively. These two eigenvalues dominate the performance of spectral methods. They will run very slowly when the gap between and is small.

In fact, the spectral methods are bipartition methods that try to split a graph into two, with a balanced size and the minimum cut. Therefore, for a network that contains multiple communities, one can find all of the communities with a hierarchical structure in a recursive way until a pre-defined stopping criterion is satisfied.

The Kernighan-Lin algorithm [21], the fast Newman algorithm [22], and the Guimera-Amaral algorithm [24] are three popular local search based optimization methods for solving NCMPs. They adopt quite a similar idea in finding a neighbor of the current solution in the problem space during each iteration, but adopt different optimization objectives and different strategies for regulating the local search process.

The Kernighan-Lin algorithm [21] (or KL for short) aims to minimize an evaluation function defined as the difference of the numbers of intra-community links and inter-community links. Starting from an initial partition of a network, in each iteration, KL moves or swaps nodes between communities in order to decrease the evaluation function. This iterative process stops when the evaluation function remains unchanged. KL runs moderately fast with the time complexity of $O\left(n^{2}\right)$. During the local search process, KL only accepts better neighbor solutions and rejects all worse ones, and thus it often finds a local, rather than a global, optimal solution. The principal restriction of $\mathrm{KL}$ is that it needs to have some prior knowledge, such as the number, as well as the average size of, communities in order to generate an initial 
partition. KL is also sensitive to initial partitions; that is, a bad one could result in a slow convergence and hence a poor solution.

Newman has presented a faster algorithm (or FN for short) for detecting community structures with the time complexity of $O(m n)$ [22]. In essence, FN is also a local search based optimization method. Starting from an initial state in which each community only contains a single node, FN repeatedly joins communities together in pairs by choosing the best merge, until only one community is left. In this bottom-up way, the dendrogram of community structure is constructed. In order to choose the best merge in each iteration, a new metric, modularity, is proposed to quantitatively measure how well-formed a community structure is. The modularity of a given network in terms of a $Q$-function is defined as follows:

$$
Q=\sum_{i} e_{i i}-a_{i}^{2}
$$

where $e_{i j}$ denotes the fraction of all weighted links in networks that connect the nodes in community $i$ to the those in community $j$, and $a_{i}=\sum_{j} e_{i j}$. It is expected that better partitions of a given network will be those with larger $Q$-values.

The algorithm proposed by Guimera and Amaral [24] (or GA for short) also tries to find a partition of a network with the maximum modularity. However, different from FN, GA adopts simulated annealing (SA) to regulate the local search process in order to obtain a better solution. Similar to KL, starting from an initial partition of a network, GA generates, evaluates, accepts or rejects a new neighbor partition from the current one in each iteration. To generate a new neighbor partition, GA moves or swaps nodes between groups, divides a group or merges two groups. Then GA evaluates the new partition by calculating its modularity and decides whether or not to accept it by using the metropolis criterion given in Eq.2 based on the current system temperature.

$$
p= \begin{cases}1 & , C_{t+1} \leq C_{t} \\ e^{-\left(C_{t+1}-C_{t}\right) / T} & , C_{t+1}>C_{t}\end{cases}
$$

where $C_{t}=-Q_{t}, p$ is the probability of accepting the solution obtained at time $t+1$, and $T$ is the system temperature at time $t+1$.

As argued by authors, GA has a good performance, due to the capability of SA, in finding a globally optimal solution. While the efficiency of GA is dominated by the convergent speed of SA, which is usually slow and very sensitive to its parameters, such as the initial layout and the strategies of finding a neighbor solution, and the cooling system temperature. GA outputs a partition of a network without a hierarchical structure, and does not require prior knowledge, such as the number of communities.

It should be noticed that it is not always safe for us to use the modularity as the optimization objective. For example, the actual partitions of some social networks correspond to locally maximum modularity values rather than global optima, as shown in Fig.2 [47]. Also, as reported by Guimera and his colleagues [33], some random networks without well defined community structures may have quite high 
modularity values due to fluctuations. Additionally, as Fortunato and Barthelemy argued [34], modularity optimization methods tend to discover coarse rather than fine ones. In those cases, the optimization based methods that maximize modularity values may not be able to find the real community structures hidden in networks.

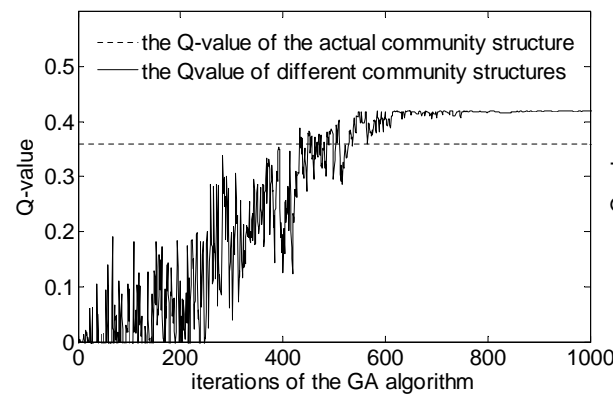

(a)

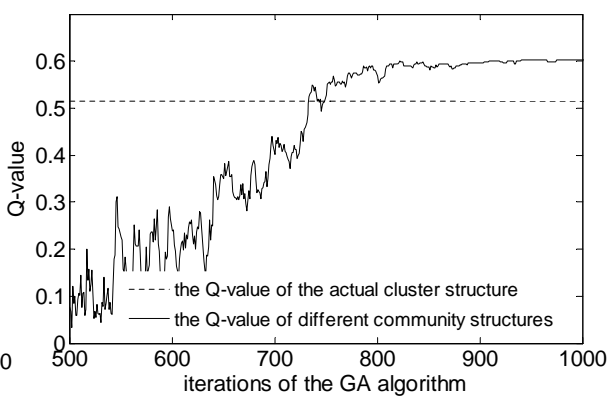

(b)

Fig. 2 The local search process of the GA algorithm [47]. In (a), GA is applied to the karate network. The detected optimal community structure consists of 4 communities, and the corresponding $Q$-value is 0.42 , which is greater than 0.37 , the $Q$-value of the actual 2-community partition of this network. In (b), GA is applied to the football association network. The detected optimal community structure contains 10 communities with a $Q$-value of 0.60 . Again, this global optimum is larger than 0.51 , the locally optimal $Q$-value of the real 12 -community structure of this network.

In the literature, there are also other optimization based algorithms for addressing NCMPs. For example, Reichardt and Bornholdt considered a network as a multiplestate Potts model, in which each node is a spin with $q$ values [35]. They suggested that the best network partition corresponds to the most stable state of the Potts model, that is, the state with the minimum energy, in which the spins with the same values constitute one community. They found such a distribution of spin values by minimizing a pre-defined energy function using a Monte Carlo optimization method combined with the simulated annealing algorithm.

\subsection{Heuristic methods}

The Maximum Flow Community (MFC) algorithm [14], the Girvan-Newman algorithm (GN) [7] and its improvements [36, 37], the Hyperlink Induced Topic Search algorithm (HITS) [13], the Wu-Huberman algorithm (WH) [28] and the Clique Percolation Method (CPM) [29] are typical heuristic based algorithms for solving NCMPs.

The MFC algorithm was proposed by Flake et al. based on the Max Flow-Min Cut theorem in graph theory [14]. The basic assumption behind is that the maximum flow through a given network is decided only by the capacity of network "bottlenecks", which is the capacity of the Min-Cut sets, and the sparse inter-community 
links can be regarded as the "bottlenecks" in the flow within the network. Therefore, the inter-community links can be discovered by calculating the Min-Cut sets. By iteratively removing "bottleneck" links, involved communities will be gradually separated from each other. Based on MFC, Flake and his colleagues proposed a method for discovering Web communities and verified an interesting hypothesis through their experiments, that is, self-organized and hyperlink-based Web community are highly topic-related [10]. So far, the best time for calculating the Min-Cut sets of a graph is $O\left(\operatorname{mnlog}\left(n^{2} / m\right)\right)[38,39]$, which decides the time of each bipartition happened in MFC. But in practice, MFC runs fast because the Min-Cut computation is restricted within a fairly small area around some pre-selected Web pages rather than the global Web.

Similarly, the GN algorithm detects all communities by recursively breaking inter-community links [7]. But, different from MFC, the heuristic rule introduced in GN is that the inter-community links are those with the maximum "edge betweenness", which is defined as the number of geodesic paths running through a given link. The GN algorithm is a hierarchical method and can produce a dendrogram of community structure in a top-down fashion. Its time complexity is $O\left(m^{2} n\right)$, which makes it not suitable for large-scale networks. In order to speed up the basic GN algorithm, several improvements have been proposed.

Tyler et al. introduced a statistical technique into the basic GN algorithm [37]. Instead of computing the exact edge betweenness of all links, they used the Monte Carlo method to estimate an approximate edge betweenness value for a selected link set. Inevitably, an improvement in speed is gained at the price of a reduction in accuracy.

Because calculating edge betweenness is time-consuming, Radicchi et al. defined a new metric, called link clustering coefficient, to replace it [36]. The assumption behind their method is that inter-community links are unlikely to belong to a short loop, such as triangles and squares; otherwise, links in the same loop would likely be across communities, and thus such links would inevitably increase the density of inter-community links. Using this heuristic rule, they define the link clustering coefficient as the number of triangles or squares in which a link is involved. In each iterative step, links with the minimum link clustering coefficient will be cut off. The average time complexity for computing the link clustering coefficient of all links is $O\left(\mathrm{~m}^{3} / \mathrm{n}^{2}\right)$, lower than that for computing edge betweenness, which is $O(m n)$. Thus, their method is on average faster than GN with the time complexity of $O\left(\mathrm{~m}^{4} / \mathrm{n}^{2}\right)$.

Similar to MFC, HITS presented by Kleinberg [13] aims to discover hyperlinkbased Web communities. The basic assumption behind HITS is that there exist authorities and hubs on the Web, and authorities are often pointed to by hubs that preferentially point to authorities. Based on the mutually reinforcing relationship between authorities and hubs, they developed an iterative method for inferring such authority-hub communities from the Web by computing the principal eigenvectors of two special matrices in terms of the adjacency matrix of the Web. A search engine based on HITS can return the most topic-related pages to users.

In the $\mathrm{WH}$ algorithm proposed by $\mathrm{Wu}$ and Huberman [28], a network is modeled as an electrical circuit by allocating one unit resistor on each link. Then, it selects 
two nodes from two distinct communities as the positive and negative poles, respectively. The assumption of the WH algorithm is that the resistance within communities will be much less than that between communities because the intra-community links are much denser than inter-community links, and thus the voltage difference of distinct communities should be more significant. Based on this heuristic, the WH algorithm can separate the group with a high voltage and the group with a low voltage from a network, by finding two maximum gaps in the node sequence sorted by their respective voltage values. Then, it determines the final division by considering the co-occurrence of nodes in such separated groups. As authors argued, their method is very fast with a linear time in terms of the size of a network. However, the WH algorithm depends heavily on its prior knowledge, which is hard to obtain beforehand. For instance, it needs to identify two "poles" belonging to different communities. Also, it needs the approximate size of each community in order to find multiple communities.

Palla and his colleagues presented CPM to discover an overlapping community structure [29]. They assumed that a network community is made of "adjacent" $k$ cliques, which share at least $k-1$ nodes with each other. Each clique uniquely belongs to one community, but cliques within different communities may share nodes. Using the above heuristic information, CPM is able to find the overlaps of communities. For a given $K, \mathrm{CPM}$ first locates all $k$ cliques $(k \leq K)$ from a given network, and then build a clique-clique overlap matrix to find out communities in terms of different $k$. But in practice, for an unknown network, it is not easy to decide what value of $k$ will result in a more reasonable community structure.

\subsection{Other methods}

Besides the above-discussed two main categories, there exist some other algorithms for solving NCMPs. For example, we can cluster a network through a bottom-up approach by repetitively joining pairs of current groups based on their similarities, such as correlation coefficients [40] and random walk similarities [41], which are defined in terms of their linkage relation. Also, we can first transform an NCMP into a clustering problem in a vector space by allocating a $\mathrm{k}$-dimensional coordinate to each node, and then cluster such spatial points using any typical spatial clustering algorithm, such as $k$-mean. Back in 1970, Hall had proposed an efficient way for transforming a network into a group of one-dimensional points by using a weighted quadratic equation in order that the nodes with dense links will be put close together, and those with sparse links will be put far away from each other [42]. Recently, Donetti and Munoz proposed a method for solving NCMPs based on quite a similar idea [43]. Their method maps a network into a $k$-dimensional vector space using the $k$ smallest eigenvectors of the Laplacian matrix before clustering spatial points. 


\section{Applications of community mining algorithms}

In this section, we will present some applications of community mining, with respect to various tasks in social network analysis.

\subsection{Network reduction}

Network reduction is an important step in analyzing social networks. In this section, we will illustrate how to reduce a complex network into a dendrogram, by means of community mining. The example discussed here is taken from the work as reported in [48], in which the network was constructed from the bibliography of the book entitled "Graph Products: Structure and Recognition [49]". The bibliography contains 360 papers written by 314 authors. Its corresponding network is a bipartite graph, in which each node denotes either one author or one paper, and link $(i, j)$ represents author $i$ publishing a paper $j$, as shown in Fig.3.

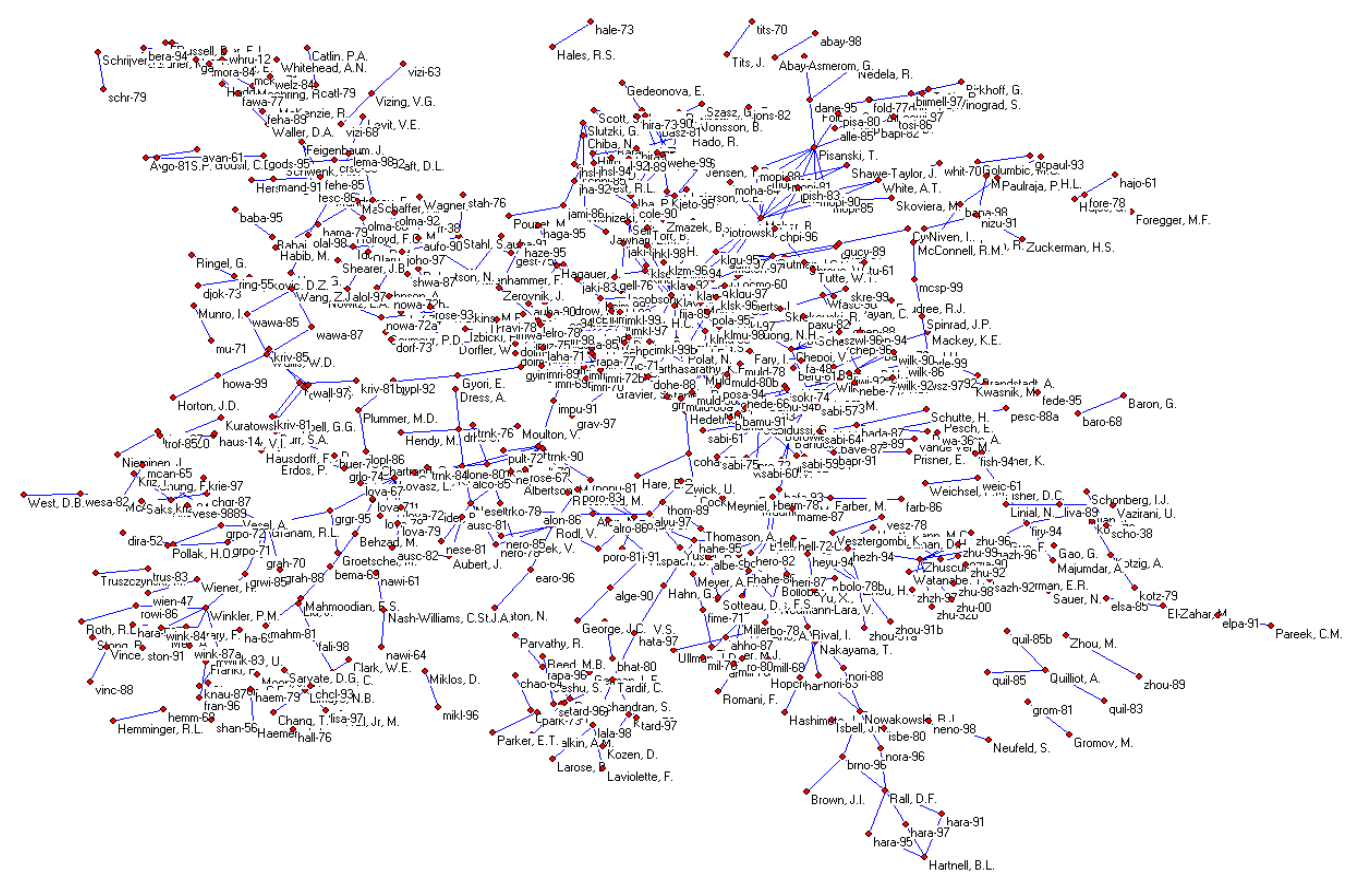

Fig. 3 The bibliography network for the book entitled "Graph Products: Structure and Recognition" [48].

Fig.4 provides the community structure as detected using a community mining algorithm, called ICS [48], in which 147 communities are uncovered from the bib- 

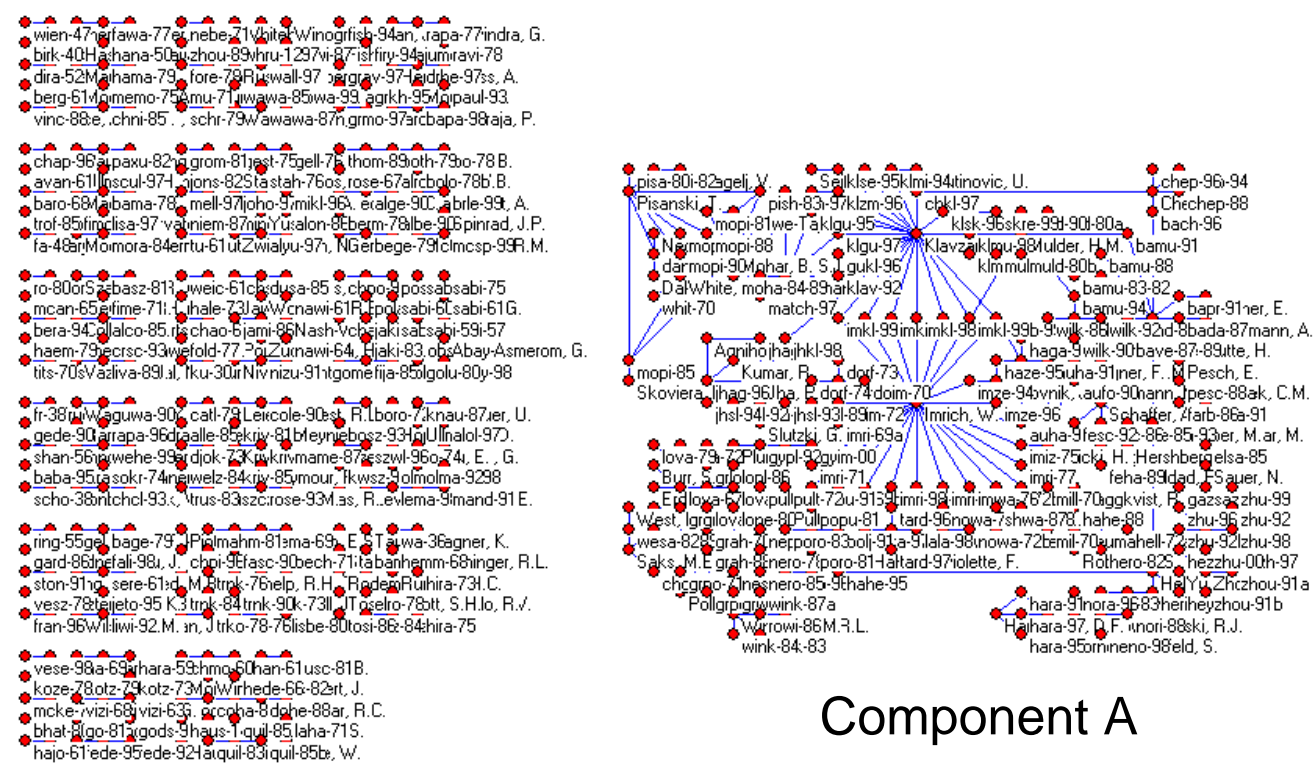

Fig. 4 The community structure of the bibliography network as detected using ICS [48].

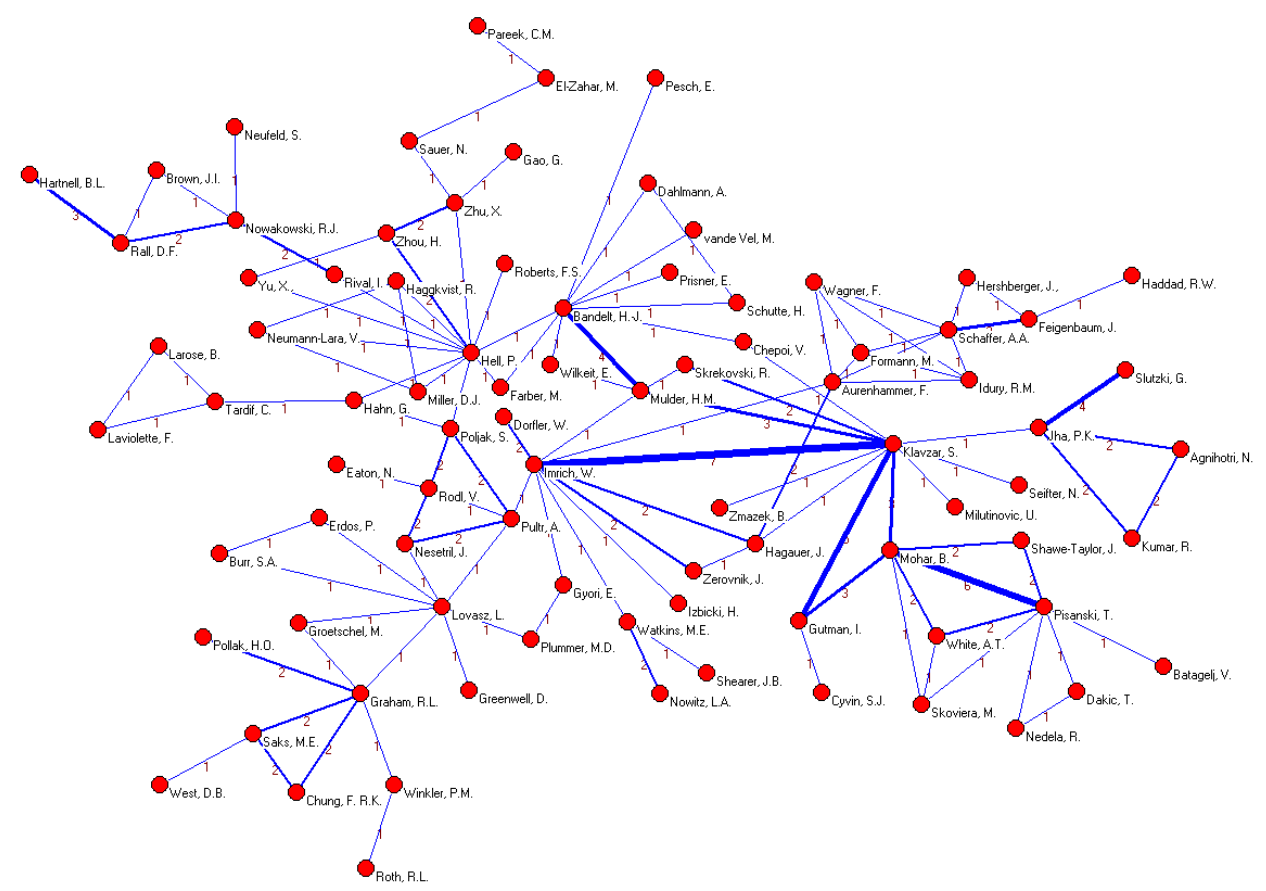

Fig. 5 The coauthor network corresponding to the biggest component [48]. 
liography network. As one would have expected, each community contains some papers and their corresponding coauthors.

Most of the detected communities are self-connected components. The component $A$ is the biggest one containing 13 communities, 158 papers, and 86 authors. Fig. 5 shows the network indicating the collaborations among these 86 coauthors, in which link $(i, j)$ with weight $w$ denotes authors $i$ and $j$ have coauthored $w$ papers. Then, ICS is again applied to the coauthor network and totally 14 communities are uncovered, as shown in Fig.6(a), in which different gray degrees indicate different communities.

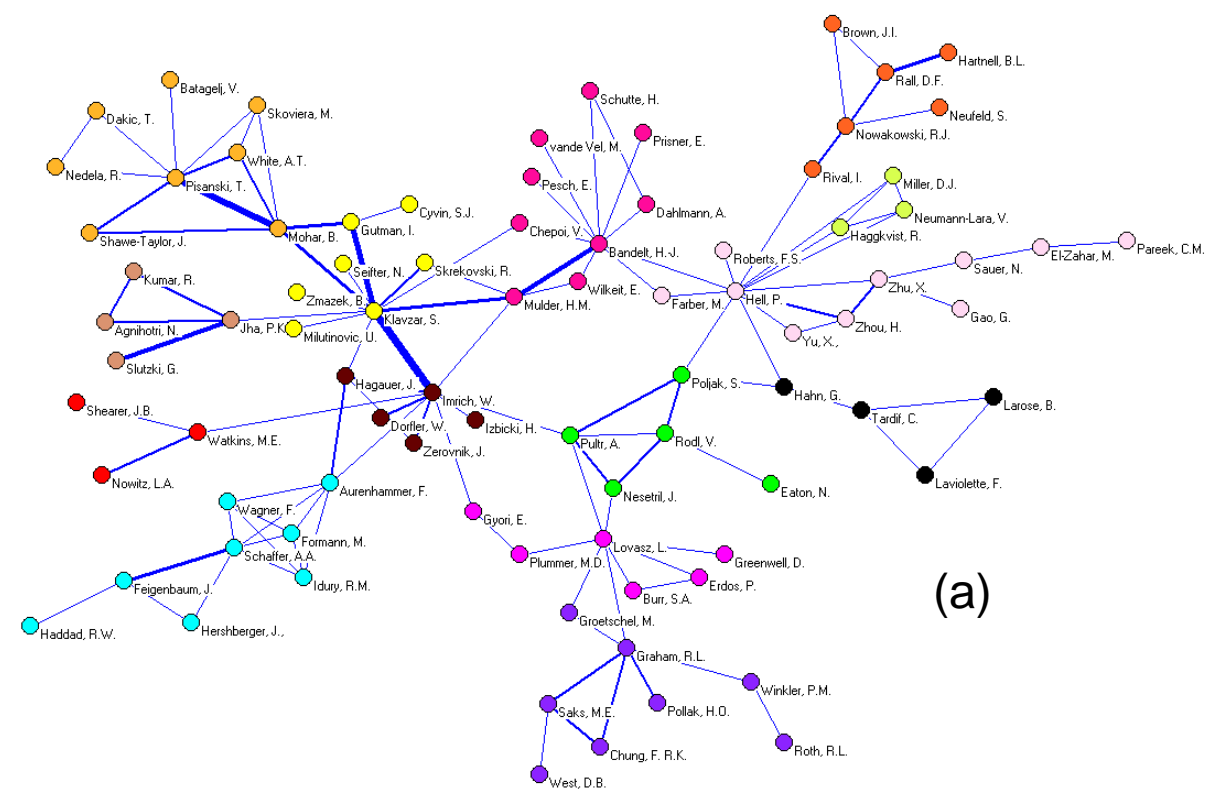

(b)

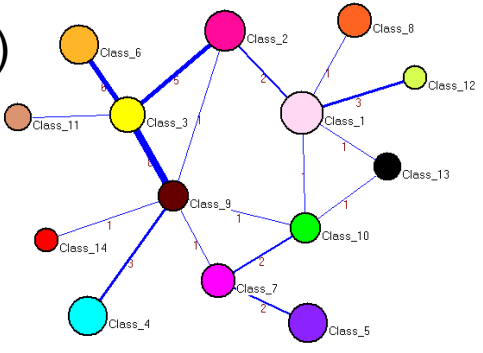

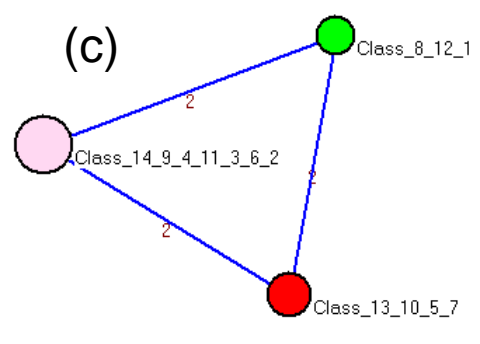

Fig. 6 The reduction of a coauthor network [48]. (a) The community structure of the network. (b) The condensed network. (c) The top-level condensed network.

Moreover, the clustered coauthor network can be reduced into a much smaller one by condensing each community as one node, as shown in Fig.6(b). Finally, the top-level condensed network corresponding to a 3-community structure is con- 


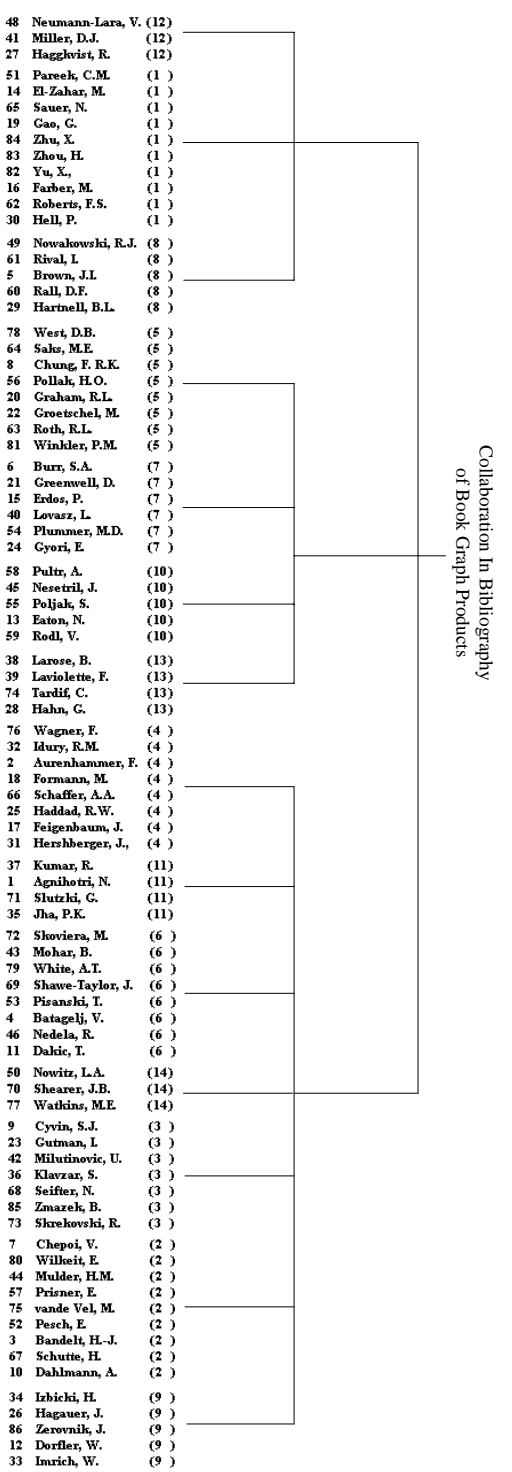

Fig. 7 The dendrogram of the coauthor network as shown in Fig.5. 
structed by using ICS from the condensed network, as shown in Fig.6(c). In this way, a dendrogram corresponding to the original coauthor network can be built, as shown in Fig.7.

\subsection{Discovering scientific collaboration groups from social networks}

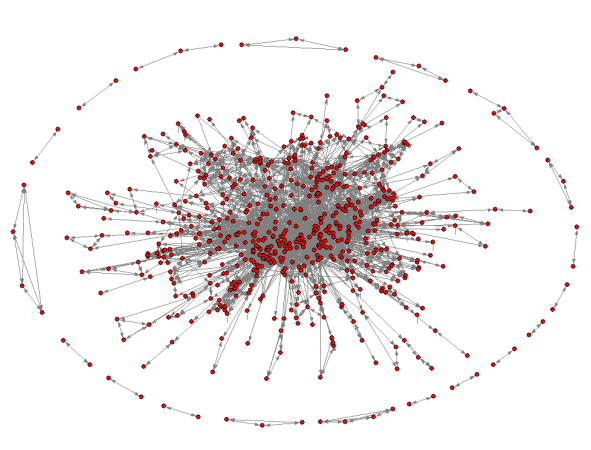

(a) The network of Flink

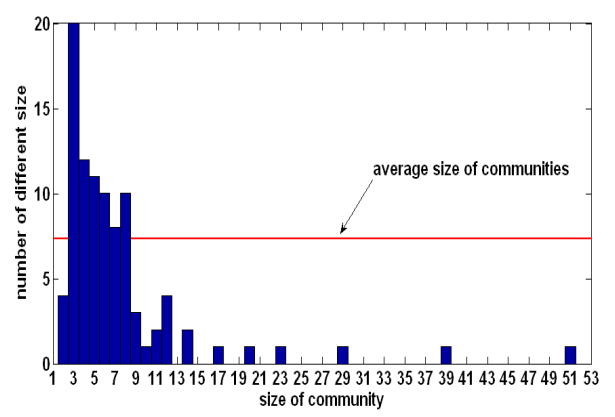

(c) The statistics of detected communities

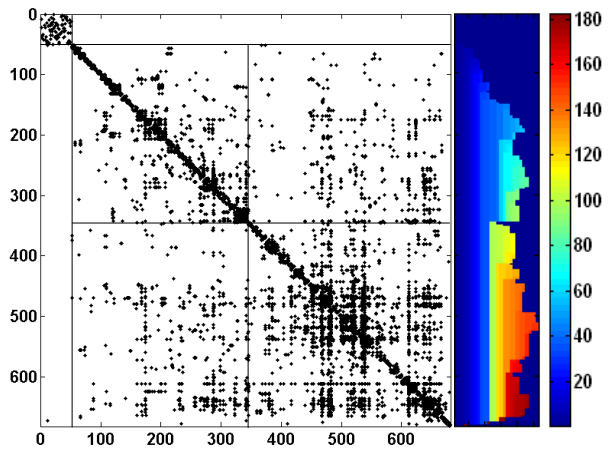

(b) The matrix with a hierarchical structure

Fig. 8 Mining a scientific collaboration network [44].

In this section, we will show how community mining techniques can be applied to the analysis of scientific collaborations among researchers using an example given in [44]. Flink is a social network that describes the scientific collaborations among 681 semantic Web researchers (http://flink.semanticweb.org/). The network was constructed based on semantic Web technologies and all related semantic information was automatically extracted from "Web-accessible information sources", 
such as "Web pages, FOAF profiles, email lists, and publication archives". Directed links between nodes, as shown in Fig.8(a), denote "know relationships", indicating scientific activities happening between researchers. The weights on the links measure the degrees of collaboration. From the perspective of social network analysis, one may be especially interested in such questions as: (i) among all researchers, which ones would more likely to collaborate with each other? (ii) what are the main reasons that bind them together? Such questions can be answered by means of applying the above-mentioned community mining techniques.

Fig.8(a) shows the network structure of Flink, while Fig.8(b) presents the output of the NCMA community algorithm proposed in [44], where each dot denotes a non-zero entry in the adjacency matrix and the gray bar at the righthand side encodes the hierarchical community structure in which different shades denote different hierarchical levels. Three biggest groups in the first level can be detected, which, respectively, contain 51, 294, and 336 members, as separated by the solid lines in the matrix. The first group is almost separated from the entire network, and its 49 members constitute the outside cycle of Fig.8(a). In total, 93 communities are detected and the average size of a community is 7.32, as shown in Fig.8(c). The selforganized communities would provide the answer to the first question. By referring to them, one can know the specific collaboration activities among these researchers. Approximately, we can observe a power-law phenomenon; most communities have a small size, while a small number of communities contain quite a large number of members.

After manually checking the profiles of members within different communities, an interesting fact has been confirmed. That is, most of communities are organized according to the locations or the research interests of their respective members. As an example, we can look into details of the largest community in which Steffen Staab is in the center, as shown in Fig.8(d). In this community, 22 of 39 come from Germany, 21 come from the same city, Karlsruhe, and 12 out of such 21 come from the same university, the University of Karlsruhe, where Steffen Staab works. Moreover, the community is research topic related; most of its members are interested in the topic related to ontology learning. These results might offer the clue to answer the second question, i.e., researchers in adjacent locations and with common interests prefer to intensively collaborate with each other.

\subsection{Mining communities from distributed and dynamic networks}

Most of the existing methods for addressing NCMPs are centralized, in the sense that they require complete information about networks to be processed. Moreover, they assume the structures of networks will not dynamically change. However, in the real world, many applications involve distributed and dynamically-evolving networks, in which resources and controls are not only decentralized but also updated frequently. In such a case, we need find a way to solve a more challenging NCMP [50]; that is to adaptively mine hidden communities from distributed and dynamic 
networks. One promising solution is based on an Autonomy-Oriented Computing (AOC) approach, in which a group of self-organizing agents are utilized. The agents will rely only on their locally acquired information about networks. In what follows, we will highlight its main ideas through an example from [50].

Intelligent Portable Digital Assistants (or iPDAs for short) that people carry around can form a distributed network, in which their users communicate with each other through calls or messages. One useful function of iPDAs would be to find and recommend new friends with common interests, or potential partners in research or business, to the users. The way to implement it will be through the following steps: (1) based on an iPDA user's communication traces, selecting individuals who have frequently contacted or been contacted with the user during a certain period of time; (2) taking the selected individuals as the input to an AOC-based [51, 52] algorithm [50], which will in turn automatically compute other members within the same community of this user in a decentralized way, by exchanging a limited number of messages with related iPDAs; (3) ranking and recommending new persons, who might not be included the current acquaintance book, the user. In such a way, people can periodically receive recommendations about friends or partners from their iPDAs.

\section{Conclusions}

In this chapter, we have introduced the network community mining problems (NCMPs), and discussed various approaches to tackling them. Generally speaking, methods for effectively and efficiently solving NCMPs can be of fundamental importance from the viewpoints of both theoretical research and practical applications. In view of their respective rationales and formulations, most of the existing methods can be classified into two categories: optimization based methods and heuristic methods.

Community mining approaches have been widely used in different areas, ranging from Web search, recommender systems, bio-informatics, to social network analysis. In this chapter, we have presented three interesting applications: network reduction, phrases clustering and distributed recommender system.

Acknowledgements This work was funded by the National Natural Science Foundation of China under Grant Nos. 60773099, 60873149 and 60973088, the National High-Tech Research and Development Plan of China under Grant Nos. 2006AA10Z245 and 2006AA10A309, the Open Project Program of the National Laboratory of Pattern Recognition (NLPR), and the basic scientific research fund of Chinese Ministry of Education under Grant No. 200903177. 


\section{References}

1. B. Krishnamurthy, "An improved min-cut algorithm for partitioning VLSI networks," IEEE Trans. on Computer, Vol.33, No. 5, 1984, pp. 438-446.

2. L. Hagen, and A.B. Kahng, "New spectral methods for ratio cut partition and clustering," IEEE Trans. on Computer-Aided Design, Vol. 11, No. 9, 1992, pp.1074-1085.

3. M.T. Heath, E.G.Y.Ng, and B.W.Peyton, "Parallel algorithm for sparse linear systems," SIAM Review, Vol. 33, 1991, pp. 420-460.

4. Pothen, H.Simon, and K.P.Liou, "Partitioning sparse matrices with eigenvalues of graphs," SIAM J. Matrix Anal. App., Vol. 11, No. 3, 1990, pp.430-452.

5. H.Simon, "Partitioning of unstructured problems for parallel processing," Computing Systems in Eng., Vol. 2, No. 3, 1991, pp. 135-148.

6. B.Hendrickson, and R.Leland, "An improved spectral graph partitioning algorithm for mapping parallel computations," SIAM J. Comp. Sci., Vol.16, No.2, 1995, pp.452-469.

7. M. Girvan and M.E.J. Newman, "Community structure in social and biological networks," Proc. of the National Academy of Science, Vol. 9, 2002, pp. 7821-7826.

8. M.E.J. Newman and M. Girvan, "Finding and Evaluating Community Structure in Networks," Phys. Rev. E, vol. 69, 2004, 026113-1 15.

9. G. Palla, A.L. Barabasi, T. Vicsek, "Quantifying social group evolution," Nature, Vol. 446, No. 7136, 2007, pp. 664-667.

10. W.Y. Chen, D. Zhang, E.Y. Chang, "Combinational Collaborative Filtering for Personalized Community Recommendation," Proc. of the 14th ACM SIGKDD International Conference on Knowledge Discovery and Data Ming (KDD’08), 2008.

11. V. Spirin, L.A. Mirny, "Protein complexes and functional modules in molecular networks," Proc. of the National Academy of Science, Vol. 100, No. 21, 2003, pp.12123.

12. D.M. Wilkinson, B.A.Huberman, "A method for finding communities of related genes," Proc. of the National Academy of Science, Vol. 101, 2004, pp. 5241-5248.

13. J.M. Kleinberg, "Authoritative sources in a hyperlinked environment," J. of ACM, Vol. 46, No. 5, 1999, pp. 604-632.

14. G.W. Flake, S. Lawrence, C.L. Giles, F.M. Coetzee, "Self-Organization and identification of Web communities," IEEE Computer, Vol. 35, No. 3, 2002, pp. 66-71.

15. H. Ino, M. Kudo, A. Nakamura, "Partitioning of Web graphs by community topology," Proc. of the 14th International Conference on World Wide Web (WWW'05), 2005, pp. 661-669.

16. M. Fiedler, "Algebraic Connectivity of Graphs," Czechoslovakian Math. J., Vol. 23, 1973, pp. 298-305.

17. M. Fiedler, "A Property of Eigenvectors of Nonnegative Symmetric Matrices and Its Application to Graph Theory," Czechoslovakian Math. J. Vol. 25, 1975, pp. 619-637.

18. A. Pothen, H. Simon, and K.P. Liou, "Partitioning Sparse Matrices with Eigenvectors of Graphs," SIAM J. of Matrix Analysis and Application, Vol. 11, 1990, pp. 430-452.

19. J.Shi, and J.Malik, "Normalized Cuts and Image Segmentation," IEEE Trans. on Pattern analysis and machine Intelligent, Vol. 22, 2000, pp. 888-904.

20. Y.C. Wei and C.K. Cheng, "Ration cut partitioning for hierarchical designs," IEEE Trans. on Computer-Aided Design, Vol. 10, No. 7, 1991, pp.911-921.

21. B.W. Kernighan, and S. Lin, "An Efficient Heuristic Procedure for Partitioning Graphs," Bell System Technical, Vol. 49, 1970, pp. 291-307.

22. M.E.J. Newman, "Fast Algorithm for Detecting Community Structure in Networks," Phys. Rev. E, Vol. 69, 2004, pp.066133.

23. Z.Wang, and J.Zhang, "In search of the biological significance of modular structures in protein networks," PLOS Comp. Bio., Vol.3, No.6, 2007, pp.e107.

24. R. Guimera, and L.A.N. Amaral, "Functional cartography of complex metabolic networks," Nature, Vol. 433, 2005, pp.895-900.

25. J.M. Pujol, J. Bejar, and J. Delgado, "Clustering algorithm for determining community structure in large networks," Phys. Rev. E, Vol. 74, 2006, 016107. 
26. M.E.J.Newman, "Modularity and community structure in networks," Proc. of the National Academy of Science, Vol. 103, No.23, 2006, pp.8577-8582.

27. J. Reichardt and S. Bornholdt, "Detecting fuzzy community structures in complex networks with a potts model," Phys. Rev. Let., Vol. 93, No. 19, 2004, pp.218701.

28. F. Wu and B.A. Huberman, "Finding Communities in Linear Time: A Physics Approach," European Phys. J. B, Vol. 38, 2004, pp. 331-338.

29. G. Palla, I.Derenyi, I.Farkas, and T.Vicsek, "Uncovering the overlapping community structures of complex networks in nature and society," Nature, Vol. 435, No. 7043, 2005, pp. 814-818.

30. B. Yang, W.K. Cheung, and J. Liu, "Community Mining from Signed Social Networks," IEEE Trans. on Knowledge and Data Engineering, Vol. 19, No. 10, 2007, pp.1333-1348.

31. M.R. Garey, and D.S. Johnson, Computers and Intractability: A Guide to the Theory of NPCompleteness, W. H. Freeman, 1979.

32. W.W. Zachary, "An Information Flow Model for Conflict and Fission in Small Groups," J. Anthropological Research, Vol. 33, 1977, pp. 452-473.

33. R. Guimera, M. Sales and L.A.N. Amaral, "Modularity from fluctuations in random graphs and complex networks," Phys. Rev. E, Vol.70, 2004, 025101.

34. S.Fortunato, M.Barthelemy, "Resolution limit in community detection," Proc. of the National Academy of Science, Vol. 104, No. 1, 2007, pp. 36-41.

35. J. Reichardt and S. Bornholdt, "Detecting fuzzy community structures in complex networks with a potts model," Phys. Rev. Let., Vol. 93, No. 19, 2004, pp. 218701.

36. F. Radicchi, C. Castellano, F. Cecconi, V. Loreto, and D. Parisi, "Defining and Identifying Communities in Networks," Proc. of the National Academy of Science, Vol. 101, No. 9, 2004, pp. 2658-2663.

37. J.R. Tyler, D.M. Wilkinson, and B.A. Huberman, "Email as Spectroscopy: Automated Discovery of Community Structure within Organizations," Proc. of the 1st International Conference on Communities and Technologies, 2003.

38. A.V. Goldberg and R.E. Tarjan, "A New Approach to the Maximum Flow Problem," J. of ACM, Vol. 35, No. 4, 1988, pp. 921-940.

39. A.V. Goldberg, "Recent Developments in Maximum Flow Algorithms," Proc. of the 6th Scandinavian Workshop on Algorithm Theory, 1998.

40. S. Wasserman and K. Faust, Social Network Analysis, Cambridge Univ. Press, 1994.

41. P. Pons, and M. Latapy, "Computing communities in large networks using random walks," J. of Graph Algorithms and Applications, Vol. 10, No. 2, 2006, pp.191-218.

42. K.M. Hall, "An r-dimensional quadratic placement algorithm," Management Science, Vol.17, No.3, 1970, 219-229.

43. L. Donetti and M.A. Munoz, "Detecting network communities: a new systematic and efficient algorithm," J. of Stat. Mech, Vol. 10, 2004, pp.P10012.

44. B. Yang, and J. Liu, "An Efficient Probabilistic Approach to Network Community Mining," Proc. of Joint Rough Set Symposium (JRS’07), 2007, pp.267-275.

45. R.L. Dobrushin, "Central limit theorem for non-stationary Markov chains, I and II," Theory Prob. Appl., Vol.1, 1956, pp. 65-80.

46. D. Lusseau, "The Emergent Properties of a Dolphin Social Network," Proc. Biol. Sci., Vol.270, No.7, 2003, pp.S186-8.

47. B. Yang, D. Liu, J. Liu, D. Jin, and H. Ma, "Complex Network Clustering Algorithms," J. of Software, Vol.20, No.1, 2008, pp.54-66.

48. B. Yang, and J. Liu, "Discovering Global Network Communities Based on Local Centralities," ACM Trans. on the Web, Vol.2, No.1, 2008, Article 9: 1-32.

49. W. Imrich and S. Klavzar, "Product Graphs: Structure and Recognition," John Wiley, New York, 2000.

50. B.Yang, J.Liu, D.Liu, ”An Autonomy-Oriented Computing Approach to Community Mining in Distributed and Dynamic Networks," Autonomous Agent Multi-Agent System, Vol.20, No.2, 2010, 123-157.

51. J.Liu, X.Jin, K.C.Tsui. Autonomy Oriented Computing. Springer/Kluwer Academic Publishers. 2004. 
52. J.Liu, X.Jin, K.C.Tsui. "Autonomy Oriented Computing (AOC): formulating computational systems with autonomous components," IEEE Trans. on Systems, Man, and Cybernetics, Part A: Systems and Humans, 2005, Vol. 35, No.6, 879- 902.

Index terms (alphabetically):

Clustering

Community mining

Social network 